\title{
Editorial
}

\section{Emerging Trends in Human ABC Transporters}

\author{
Toshihisa Ishikawa ${ }^{1,2}$
}

Received December 8, 2008; accepted December 9, 2008; published online December 24, 2008

Evidence is accumulating to strongly suggest that drug transporters are amongst the determining factors governing pharmacokinetic profiles of drugs and, thus their overall pharmacological effects. A variety of drug transporter genes have been cloned and classified as either solute carriers (SLC) or ATP-binding cassette $(\mathrm{ABC})$ transporters. Such drug transporters are expressed in various tissues such as the intestine, brain, liver, kidney, and more importantly in cancer cells where they play critical roles in the absorption, distribution, and excretion of drugs. For example, transporter proteins expressed in epithelial cells of the small intestine and in brain capillary endothelial cells, greatly affect the oral bioavailability of drugs and their penetration into the central nervous system (CNS).

In this theme issue, Xia-Yan Chu et al. of Dr. Raymond Evers' group (DMPK Global Technologies, Merck \& Co.) reported that the metabolism and renal elimination of gaboxadol in humans. In particular, they addressed the role of UDP-glucuronosyltransferases UGT1A6, UGT1A7, and UGT1A8 as well as drug transporters, including hOAT1, hOAT3, hOCT2, MRP2 (ABCC2) and MRP4 (ABCC4). Gaboxadol is a selective extrasynaptic agonist of the $\gamma$-aminobutyric acid type $\mathrm{A}\left(\mathrm{GABA}_{\mathrm{A}}\right)$ receptor and is excreted in humans into the urine as parent drug and glucuronide conjugate. They examined the contribution of the above-mentioned drug metabolizing enzymes and transporters to metabolism and renal excretion.

Recently high-speed functional screening and quantitative structure-activity relationship analysis methods were developed to study the substrate/inhibitor specificity of drug transporters. In addition, the transporter-knock out mice provides a useful tool to evaluate the contribution of drug transporters to pharmacokinetic profiles of drugs and to drug-drug interactions. Suneet Shilka et al. in Dr. Suresh V. Ambudkar laboratory (National Cancer Institute, $\mathrm{NIH})$ evaluated in vivo efficacy of curcumin as an inhibitor of $\mathrm{ABC}$ transporters. They used photoaffinity labeling technology to characterize the interaction between sulfasalazine and human $\mathrm{ABC}$ transporter ABCG2. The inhibitory effect of curcumin on ABCG2 was evaluated in brain capillaries from rats. In their paper, by

\footnotetext{
${ }^{1}$ Tokyo Institute of Technology, Nagatsuta, Yokohama, Japan.

${ }^{2}$ To whom correspondence should be addressed. (e-mail: tishikaw@ bio.titech.ac.jp)
}

using Abcg2-knock out mice, they provided first evidence for the inhibition by curcumin of ABCG2-mediated efflux sulfasalazine.

Ran An et al. in Dr. Toshihisa Ishikawa laboratory (Tokyo Institute of Technology) developed a high-speed screening method for detection of drug-induced photosensitivity in vitro and evaluated the impact on photo-sensitivity of the inhibition by cyclin-dependent kinase (CDK) inhibitors on ABCG2 function. ABCG2 plays a pivotal role in the cellular homeostasis of porphyrins and the limitation of phototoxic porphyrins in the small intestine. Based on high-speed screening, QSAR analysis, and ab initio molecular orbital calculation results, they suggested that planar structure is an important factor for interactions with the active site of ABCG2. In addition, they also provided a new approach to studying drug-induced phototoxicity in vitro.

Pharmacogenomics, on the other hand, is the study of the influence of genetic factors on drug action. This research field is becoming increasingly important for predicting pharmacokinetics profiles and/or adverse reactions to drugs. Single nucleotide polymorphisms (SNPs) of the $A B C G 2$ gene, for example, have been suggested to be a significant factor in patients' responses to medication and/or the risk of diseases. In this theme issue, Tomomi Furukawa et al. in Dr. Ishikawa's laboratory (Tokyo Institute of Technology) evaluated the impact of the major nonsynonymous SNP Q141K on lysosomal and proteasomal degradations. Evidence is provided to show that the SNP Q141K affects the stability of the ABCG2 protein in the endoplasmic reticulum (ER) and enhances its susceptibility to ubiquitin-mediated proteasomal degradation. Their paper provides a new insight into the impact of genetic polymorphisms on the endoplasmic reticulumassociated degradation (ERAD) of $\mathrm{ABC}$ transporters.

\section{INTERVIEW}

1. What do you think holds the key to your success as a pharmaceutical scientist?

Actually, I am not originally a pharmaceutical scientist, because I obtained my Ph.D. degree for biophysics and biochemistry research from the Graduate School of Science at Hokkaido University. However, since I had a 5-year experience in the pharmaceutical industry (i.e., Pfizer, Inc.), I can give some ideas or hints based on my experiences to young pharmaceutical scientists. I think it is critically important to 
continuously maintain the self-motivation in terms of creating original and unique ideas. In addition, it is also important to focus one's best effort to achieve their goal. When I started my research on ATP-dependent transport of glutathione conjugates in 1982, only a few people were interested in my research subject. To me, therefore, it was critically important to keep my research going and to provide evidence for the physiological importance of the ATP-dependent transporter in order to convince many other scientists. For over more than twenty years, I have been trying to create new ideas and to develop new methods, as described below.

2. What do you consider to be your key research accomplishments?

The first key accomplishment is that I provided evidence for the existence of an ATP-dependent transport system for glutathione disulfide and glutathione conjugates in the cardiac tissue. It was in 1984 that we had first reported our findings in J. Biol. Chem. 259(6):3838-3843, a long time before the discovery of ABC transporter genes. Thereafter, by using different technologies, such as in vivo NMR and plasma membrane vesicles prepared from rat hearts, the transport of those compounds was found to be ATP-dependent primary active membrane transport. Furthermore, we have provided evidence that cysteinyl leukotriens are endogenous substrates for the ATP-dependent transporter (J. Biol. Chem. 264 (29):17343-17348, 1989; J. Biol. Chem. 265(31):19279-19286, 1990). Just after I moved from German Cancer Research Center in Heidelberg, Germany to M.D. Anderson Cancer Center in Houston, Texas, USA, I published a review article in Trends Biochem. Sci. 17(11):463-468, 1992 to propose the new concept of the "Phase III" system in xenobiotic metabolism.

The second key accomplishment is the development of high-speed screening and QSAR analysis methods to evaluate the function of ABC transporters, genetic polymorphisms and drug-drug interactions (Methods Enzymol. 400:485-510, 2005). These new technologies enabled us to quantitatively analyze the substrate specificity of human ABC transporters and to design new anticancer drugs that can circumvent multidrug resistance of cancer (Mini Rev. Med. Chem.7: 10091018, 2007). In addition, in collaboration with Prof. Kathleen Giacomini (University of California San Francisco), Dr. Shiew-Mei Huang (FDA), and Dr. Donald Tweedie (Boehringer Ingelheim), we established the International Working Group to standardize the in vitro assay methods for human drug transporters. Most recently (October 2 and 3, 2008), we organized the FDA Critical Path Transporter Workshop in Bethesda, MD to prepare a White Paper for FDA and the pharmaceutical industry.

\section{What was turning point in your career?}

My career is very unique as indicated in my biographical sketch: I obtained the Ph.D. degree of Science (Biophysics and Biochemistry) in Japan and then worked for 7 years in Germany, for 5 years in USA, and for 5 years in the pharmaceutical industry (Pfizer, Inc.). In June 2000, I came back to the academia to continue my career as Professor at Tokyo Institute of Technology. There were at least two major turning points in my career, namely, when I became Assistant Professor at University of Texas M.D. Anderson Cancer
Center in 1991 and Manager at Pfizer Inc. Central Research in Japan, 1995. At M. D. Anderson Cancer Center, I learned the importance of translational research that bridges a gap between basic science and clinical practice. I am not medical doctor, and therefore I am unable to do any clinical treatments for cancer patients. However, I started to think about possible applications of our knowledge for patient care in clinical situations. In April 1995, I was informed that my father had lung cancer. Indeed, his situation was very critical. I immediately closed my laboratory at M.D. Anderson Cancer Center and left for Japan to take care of my father. One month after I had returned to Japan, my father died. At that time, I could not get any academic position in Japan but found a job at the Central Research of Pfizer Inc. Japan. While the drug discovery research in the pharmaceutical industry greatly differed from the basic research experienced in academia, I tried to study hard the strategy of drug discovery and development, patent application, clinical research, and market evaluation. This experience I had in Pfizer, Inc. has become an extremely important turning point in my career.

4. Who are the individuals who had the greatest influence on your research career?

Hitherto, I met many people who influenced my career. In particular, Professors Helmut Sies (Düsseldorf University, Germany), Alton Meister (Cornel University Medical School, USA), and Ryoji Noyori (RIKEN, Nobel Prize laureate for Chemistry) are the individuals who were the most influential on my life and career. Prof. Sies was my first boss when I worked as Post-doctoral fellow in Düsseldorf, Germany. From him I could learn a lot of fundamentality important subjects with respect to biochemistry, physiology, pharmacology, and also the philosophy required for a scientist. Actually, in his laboratory, I could create that new idea on ATPdependent transport of glutathione disulfide and glutathione conjugates in the heart under oxidative stress.

Prof. Meister was a giant in the research field of glutathione metabolism. In the beginning, he did not agree with my idea about ATP-dependent transport of glutathione disulfide in the heart. However, he as an Associate Editor carefully checked my manuscript and corrected my grammatical failures when I submitted my single-authored paper to Journal of Biological Chemistry in 1989. I guess he finally noticed the physiological importance of ATP-dependent transport of leukotriene $\mathrm{C}_{4}$ addressed in my paper. As thus, this paper became a landmark in my career.

My collaboration with Prof. Noyori was very successful, when I was working at M.D. Anderson Cancer Center in Houston, USA. We discovered that anticancer prostaglandins chemically synthesized in his laboratory were very potent in inducing p21 cycline-dependent kinase inhibitor in a p53independent manner and arrested the cell cycle of cancer cells in G1 phase (Biochem. J. 336(3):569-576, 1998). I was so strongly impressed with by the "beauty" of small synthetic molecules that I decided to enter the pharmaceutical research field. Thereafter, he kindly wrote a strong recommendation letter for me when I applied for the present position of Professor at Tokyo Institute of Technology in 2000. In the following year, Prof. Noyori received the Nobel Prize for Chemistry. 
5. Pharmaceutical scientists are faced with the dilemma of having to publish in biomedical or basic science journals. Does it mean cutting edge science will not likely be featured in the Pharmaceutical Research?

This is a difficult question to answer. However, I believe that Pharmaceutical Research has its own editorial philosophy that is distinct form other biomedical or basic science journals. There is no doubt in the fact that the field of pharmaceutical research is indispensable to bridge a gap between basic science and clinical applications. As long as pharmaceutical scientists keep creating new and original ideas, they not need to worry about the dilemma of having to publish in biomedical or basic science journals.

6. Where is the field of $\mathrm{ABC}$ transporters going, and how do articles in the theme section fill up?

During the past two decades, research on ABC transporters evolved enormously. In the 1980s, cDNA encoding human P-glycoprotein/MDR1 (ABCB1) was first cloned and a lot of studies were carried out to characterize the molecular properties and biological significance of that ABC transporter. Since the discovery of the ABCB1 gene, many of human $\mathrm{ABC}$ transporters including MRPs (ABCCs) and BCRP (ABCG2) were discovered and characterized.

In the next upcoming decade, I believe that our efforts and interests should be directed toward the application of knowledge of human $\mathrm{ABC}$ transporters to clinical practice and drug discovery/development. Human ABC transporters are expressed in various tissues such as the intestine, brain, liver, kidney, and importantly cancer cells where they play critical roles in the absorption, distribution, and excretion of drugs. In particular, ABC transporter proteins expressed in epithelial cells of the small intestine and in brain capillary endothelial cells, greatly affect the oral bioavailability of drugs and their penetration into the central nervous system (CNS). Pharmacogenomics of human ABC transporters should be one of the most important challenges to realize personalized medicine. This theme section provides a new insight into the coupling of drug metabolism and drug transport, the importance of ABC transporters in drug safety, and the impact of genetic polymorphisms on protein expression level of $\mathrm{ABC}$ transporters.

7. What are the challenges for $\mathrm{ABC}$ transporters and how can they be overcome?

It is our important mission and challenges to apply our knowledge of human $\mathrm{ABC}$ transporters to clinical practice and drug discovery/development. Genetic polymorphisms of human ABC transporter genes are reportedly related to the risk of diseases and patients' responses to medication. Development of SNP typing methods, that are rapid, accurate, and lowly priced, would provide a practical approach to examine a latent link between genetic polymorphisms of human ABC transporter genes and pharmacokinetics profiles of drugs. In this context, we are currently developing the world's-fastest SNP detection system for clinical practice.
8. What is the key to developing successful collaborative relationship?

"Trust" is the keyword that will lead us to a successful collaborative relationship. In my laboratory, we have a multidisciplinary collaboration platform consisting of a variety of companies with specific expertise, e.g., construction of medical instruments or optical analysis systems, information technology, reagent production, and chemical synthesis. In addition, collaboration with academic scientists runs very frequently in my laboratory. At present, we are developing a medical engineering network in Japan to establish the infrastructure of personalized medicine for cancer chemotherapy. In such collaborations, "trust" is of utmost importance, and this mutual trust enhances the motivation amongst collaborators.

9. Where is the place for collaboration with industry in academia?

Since I have worked in the pharmaceutical industry for 5 years, I am aware of many critical points currently residing in the R\&D operation of drug discovery and development. Therefore, we are developing new technologies, such as highspeed screening for functional analysis of drug transporters and QSAR analysis methods for drug molecular design. These technologies are immediately applicable to the R\&D operation of drug discovery and development. In other words, pharmaceutical companies are "customers" in my research. The goal of my research laboratory, therefore, is to develop our original methods and to support drug discovery and clinical diagnosis.

10. What is your philosophy of educating graduate students?

My philosophy of educating graduate students is very simple: "Science must be for the people and for the society". As long as we are working in the academia field, we should keep it in mind that our salary as well as research money are paid from the tax money of people and industry. Therefore, throughout our education and research, we are responsible to create scientific "fruits" for people and to feedback these benefits to the society. In this context, I do not fully agree with the current situation in which many basic and biomedical scientists are seeking for research subjects with high impact factors. Impact factors do not reflect the benefit on the society at all!

11. What are the challenges facing the pharmaceutical sciences?

In the post-genome-sequencing era, emerging genomic technologies resulting in paradigm shifts for drug discovery and development. Nevertheless, drug discovery and development still remain high-risk, high-stakes ventures with long and costly timelines. Indeed, the attrition of drug candidates in preclinical and developmental stages is a major problem in drug design. In that light, a transport mechanism-based design strategy might help to create new, pharmacokinetically advantageous drugs, and such challenges should be considered as an important component of drug design strategy in pharmaceutical science. 


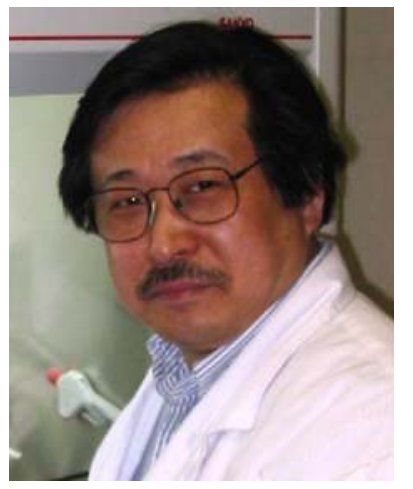

Dr. Ishikawa obtained his Ph.D. degree from the Graduate School of Science, Hokkaido University, Japan in March 1982. In the same year, he received the scholarship of the Deutscher Akademischer Austauschdienst (DAAD) and moved to Germany. From 1982 till 1987, he was a postdoctoral fellow at the Institute of Physiological Chemistry (Prof. Helmut Sies), Medical School of University Düsseldorf, in Germany. In April 1987 he returned to Japan and was appointed Assistant Biochemist at the Department of Biochemistry, Medical School of Osaka University, Japan. In 1989, he went to Germany again to become the project leader at the Department of Tumor Biochemistry in German Cancer Research Institute (DKFZ), Heidelberg. In 1991, Dr. Ishikawa left Germany for U.S.A, as he was appointed Assistant Professor at the Department of Experimental Pediatrics, University of Texas M.D. Anderson Cancer Center in Huston, Texas, U.S.A. In Houston, he had an adjunct appointment as Assistant Professor at the Graduate School of Health Science Center, University of Texas. In 1993, he received the
Achievement Award from the International Life Sciences Institute (Washington DC). In December 1995, he was appointed to Senior Scientist and Manager at the Department of Medicinal Biology in the Central Research of Pfizer, Inc. and thereafter he became the Director of the Department of Research Technology Development at the Japanese Headquarters of Pfizer, Inc. in Tokyo, 1999. Since June 1, 2000, Dr. Ishikawa is a Professor at the Graduate School of Bioscience and Biotechnology, Tokyo Institute of Technology, Japan. In 2003, he founded a bio-venture company named "MedicinalGenomix, Inc.". He is also Professor (adjunct) at the Graduate School of Tokyo Medical and Dental University as well as Senior Guest Scientist (adjunct) at the Omics Research Center of RIKEN Yokohama Institute. Dr. Ishikawa was a member of the International Nomenclature Committee for Human ABC Transporter Genes. He directed the NEDO International Collaboration Research project entitled "International standardization of in-vitro functional assay methods for human drug transporters" in 2005-2008. He received the Research Award for his distinguished study on "Pharmacogenomics of human ABC transporters" as well as the Fellow Degree from the Japanese Society for Study of Xenobiotics (JSSX) in 2005 and 2008 , respectively. In addition, Dr. Ishikawa is currently serving as Associate Editor for several journals including Journal of Experimental Therapeutics and Oncology and Pharmacogenomics. In 2004, Dr. Ishikawa organized the 24th International Symposium on "Pharmacogenomics in Cancer Chemotherapy: Recent Advances in ABC Transporters and Genome Analyses" in Sapporo, Japan. Most recently, he was one of the Steering Committee members to organize the FDA Critical Path Transporter Workshop held in Bethesda, MD on October 2-3, 2008. Thus, Dr. Ishikawa has become an internationally-recognized scientist in the research field on pharmacogenomics of human $\mathrm{ABC}$ transporter genes. In addition, he is also currently developing the world's-fastest SNP detection system for clinical practice. 